\title{
Zero-Shear Viscosity of Block Copolymers in Semidilute Solutions
}

\author{
Masayoshi Yamaguchi, Norihisa Maeda,* Yoshiaki TaKahashi, \\ Yushu Matsushita, and Ichiro NoDA \\ Department of Synthetic Chemistry, Nagoya University, Furo-cho, \\ Chikusa-ku, Nagoya 464-01, Japan
}

(Received August 30, 1990)

\begin{abstract}
The zero-shear viscosity $\eta^{0}$ of styrene-2-vinylpyridine diblock copolymers in semidilute solutions was measured in pyridine (a commonly good solvent), methyl ethyl ketone (MEK) (a commonly poor solvent) at $25^{\circ} \mathrm{C}$, and in benzene (a selective solvent) at $11.4^{\circ} \mathrm{C}$. It was found that the reduced zero-shear viscosity $\eta_{\mathrm{R}}^{0}\left(\equiv \eta_{\mathrm{sp}}^{0} / C[\eta]\right)$ is expressed as a universal function of $C[\eta]$ in all solvents, and the dependence of $\eta_{\mathrm{R}}^{\mathrm{o}}$ on $C[\eta]$ is determined by the exponent in the relationship between the radius of gyration and molecular weight of the block component which has the larger excluded volume, regardless of the solvent.

KEY WORDS Zero-Shear Viscosity / Block Copolymer / Scaling Theory / Solvent Effect /
\end{abstract}

The zero-shear viscosity $\eta^{0}$ of homopolymer solutions is discussed by classifying solutions into at least three concentration regions in terms of concentration $C$ and degree of coil-overlapping $C / C^{*} .^{1,2}$ Here, the critical concentration $C^{*}$, at which polymer chains begin to overlap with each other can be defined by

$$
C^{*}=3 M /\left(4 \pi\left\langle s^{2}\right\rangle^{3 / 2} N_{\Lambda}\right)
$$

where $M$ is the molecular weight, $\left\langle s^{2}\right\rangle$ is the mean square radius of gyration and $N_{A}$ is Avogadro's number.

In dilute solutions where $C \ll 1$ and $C / C^{*} \ll 1$, the reduced zero-shear viscosity $\eta_{\mathbf{R}}^{0}$ is given by

$$
\begin{aligned}
\eta_{\mathrm{R}}^{0} \equiv \eta_{\mathrm{sp}}^{0} / C[\eta] & =1+k^{\prime} C[\eta]+\cdots \\
& =1+k C / C^{*}+\cdots
\end{aligned}
$$

where $\eta_{\mathrm{sp}}^{0}=\left(\eta^{0}-\eta_{\mathrm{s}}\right) / \eta_{\mathrm{s}}, \quad \eta_{\mathrm{s}}$ is the solvent viscosity, $[\eta]$ is the intrinsic viscosity, $k^{\prime}$ is the Huggins' constant, and $k=3 k^{\prime} \Phi^{\prime} /\left(4 \pi N_{\mathrm{A}}\right)$. Here, the last equation is obtained by assuming the Flory-Fox equation, $[\eta]=\Phi^{\prime}\left\langle s^{2}\right\rangle^{3 / 2} / M$ where
$\Phi^{\prime}$ is the Flory viscosity factor. ${ }^{3}$

In semidilute solutions where $C \ll 1$, but $C / C^{*} \gg 1, \eta_{\mathrm{R}}^{0}$ is given by a scaling law as ${ }^{4-6}$

$$
\eta_{\mathrm{R}}^{0} \alpha\left(C / C^{*}\right)^{(4,4-3 v) /(3 v-1)} \propto(C[\eta])^{(4,4-3 v) /(3 v-1)}
$$

where $v$ is the exponent in the relationship between radius of gyration and molecular weight, $\left\langle s^{2}\right\rangle \propto M^{2 v}$. Here, the second equation is also derived assuming the Flory-Fox equation. Thus, $\eta_{\mathrm{R}}^{0}$ is predicted to be expressed as a universal function of $\left(C / C^{*}\right)$ or $C[\eta]$ in both the dilute and semidilute regions, and this theoretical prediction was confirmed by experiments as reported previously. ${ }^{5,6}$

In block copolymer solutions, microphase separation occurs above a critical concentration even in commonly good solvents. ${ }^{7}$ Particularly, in selective solvents which dissolve a block chain, but not others, block copolymers form a strong three-dimensional macrolattic in solutions. Thus, viscosities of block copolymer solutions have been studied mainly in relation

* Present address: KANEBO-NSC, Ltd., 1-6-5 Senbanishi, Minoo, Osaka 562, Japan. 
to the microphase-separated structures. ${ }^{8,9}$

Measurements of viscosity, light scattering and small-angle neutron scattering revealed that a block copolymer does not assume intramolecular segregation and behaves like a homopolymer in dilute solutions. ${ }^{10-13}$ However, solution properties of block copolymers from dilute to microphase-separated regions are little known. In the present work, therefore, we measured $\eta^{0}$ of block copolymers in the intermediate or semidilute region between the dilute and microphase-separated regions to examine the applicability of the scaling law (eq 3 ), which is valid for homopolymers in semidilute solutions, ${ }^{5,6}$ to $\eta^{0}$ of block copolymer solutions.

\section{EXPERIMENTAL}

\section{Samples}

Samples used were styrene-2-vinylpyridine (SP) diblock copolymers, which were prepared in vacuo at $-78^{\circ} \mathrm{C}$ by an anionic polymerization method with a sequential monomer addition technique. Details of the preparation and characterization of the block copolymers are reported in previous papers. ${ }^{13,14}$ The molecular characteristics of samples are listed in Table I.

\section{Solvents}

Solvents used were pyridine, methyl ethyl ketone (MEK) and benzene. Pyridine and MEK of the specific grade of Kishida Chemical Co., were dried according to standard procedure. Benzene of the spectroscopic grade of Merck was used without further purification. Pyridine is a good solvent for both polystyrene (PS) (0.73) and poly(2-vinylpyridine) (P2VP) (0.73), MEK is a poor solvent for both PS (0.63) and P2VP (0.48), while benzene is a selective solvent which is good for PS $(0.70)$ but as a $\theta$-solvent for P2VP (0.5) at $11.4^{\circ} \mathrm{C}$. Here, the values in parentheses denote the exponent $\mathrm{a}$ in the Mark-Houwink-Sakurada (MHS) equation, $[\eta]=K M^{\mathrm{a}}$ for PS and P2VP
Table I. Molecular characteristics of styrene-2vinylpyridine diblock copolymers

\begin{tabular}{cccc}
\hline Sample code & $M_{w} \times 10^{-5}$ & $M_{w} / M_{n}{ }^{\mathrm{a}}$ & $C_{\mathrm{S}} / \mathrm{wt} \%^{\mathrm{b}}$ \\
\hline SP-12 & 4.22 & 1.03 & 50 \\
SP-23 & 10.9 & 1.03 & 51 \\
SP-18 & 15.6 & 1.17 & 48 \\
\hline
\end{tabular}

a $M_{w} / M_{n}$ were determined from GPC.

b $C_{\mathrm{s}}$ denotes styrene content.

Table II. Solvent viscosity

\begin{tabular}{cccc}
\hline & \multicolumn{3}{c}{$\eta_{\mathrm{s}} / \mathrm{mPa} \mathrm{s}$} \\
\cline { 2 - 4 } Temp $/{ }^{\circ} \mathrm{C}$ & Pyridine & MEK & Benzene \\
\cline { 2 - 4 } & & & 0.736 \\
25.0 & 0.884 & 0.378 & \\
\hline
\end{tabular}

in the respective solvents. ${ }^{13}$ The viscosities of these solvents $\eta_{\mathrm{s}}$ are listed in Table II.

\section{Zero-Shear Viscosity Measurements}

Weighed amounts of polymer sample and solvent were mixed, and the solutions were kept at about $40^{\circ} \mathrm{C}$ in an incubator for two weeks to be dissolved completely. The concentrations were converted to $\mathrm{g} \mathrm{cm}^{-3}$ by assuming the additivity of specific volumes of polymer and solvent. The zero-shear viscosity $\eta^{0}$ was measured with capillary viscometers of MaronKrieger-Sisko type ${ }^{15}$ and the Ubbelohde type in pyridine and MEK at $25^{\circ} \mathrm{C}$, and in benzene at $11.4^{\circ} \mathrm{C}$. The $\mathrm{MHS}$ equation of the diblock copolymer in benzene at $11.4^{\circ} \mathrm{C}$ was determined in this work.

\section{RESULTS AND DISCUSSION}

No shear-rate dependence of viscosity as reported in microphase-separated regions ${ }^{8,9}$ was observed for any sample solution measured in this work. Thus, the effects of microphase separation on the viscosity were not observed explicitly.

Figures $1-3$ show double logarithmic plots of $\eta_{\mathrm{sp}}^{0} v s . C$ in pyridine, MEK and benzene, 
Zero-Shear Viscosity of Block Copolymers in Semidilute Solutions

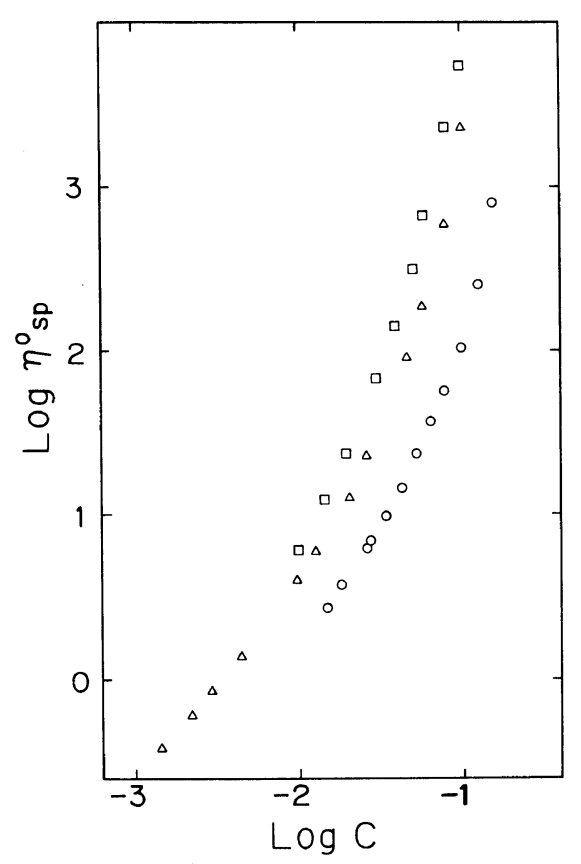

Figure 1. Concentration dependence of specific viscosity $\eta_{\mathrm{sp}}^{0}$ in pyridine at $25^{\circ} \mathrm{C}$. Symbols $(\bigcirc),(\triangle)$, and $(\square)$ denote the data for samples SP-12, SP-23 and SP-18, respectively.

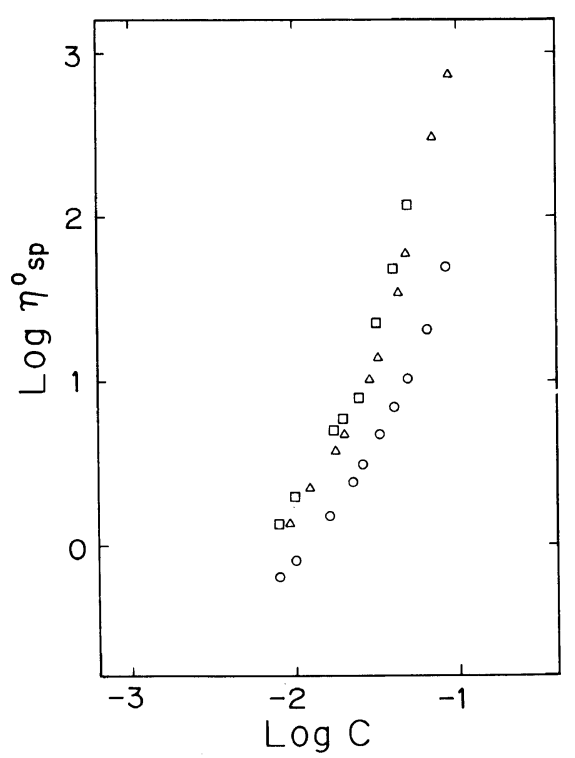

Figure 2. Concentration dependence of specific viscosity $\eta_{\mathrm{sp}}^{0}$ in MEK at $25^{\circ} \mathrm{C}$. Symbols are the same as in Figure 1.

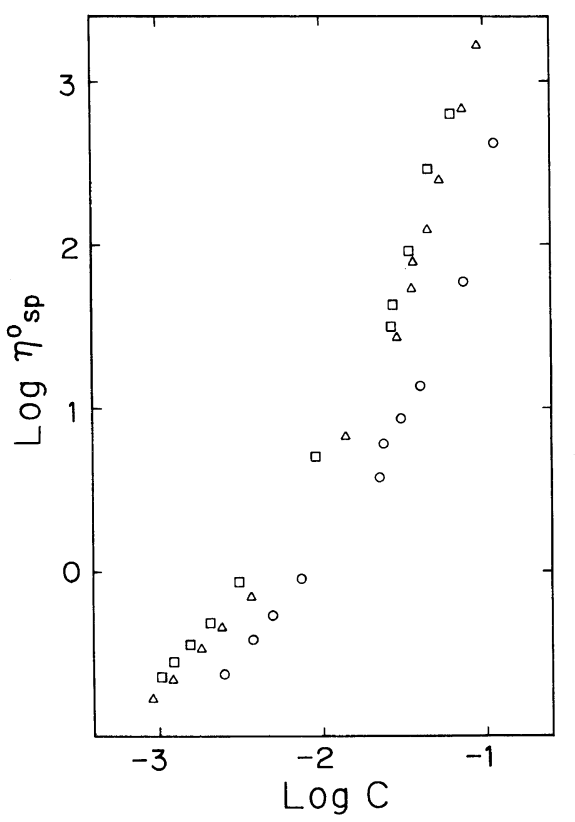

Figure 3. Concentration dependence of specific viscosity $\eta_{\mathrm{sp}}^{0}$ in benzene at $11.4^{\circ} \mathrm{C}$. Symbols are the same as in Figure 1.

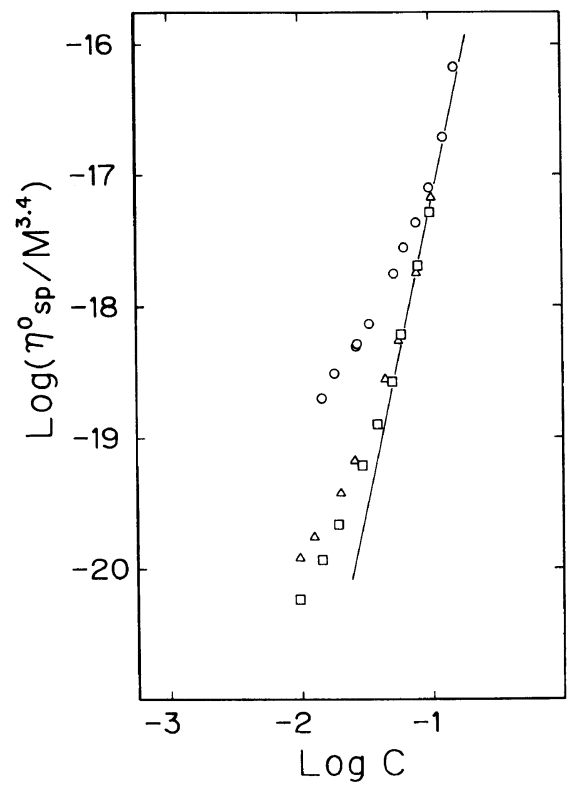

Figure 4. Double logarithmic plots of $\eta_{\mathrm{sp}}^{0} / M^{3,4} v s . C$ in pyridine. Symbols are the same as in Figure 1. 


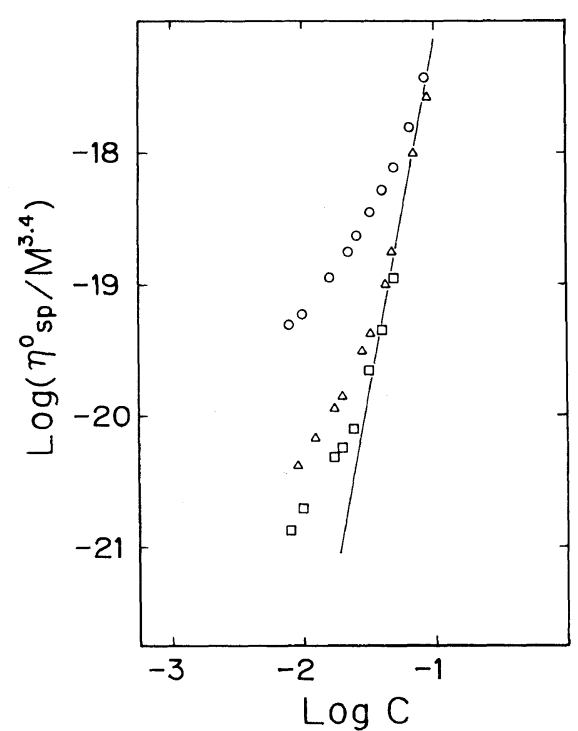

Figure 5. Double logarithmic plots of $\eta_{\mathrm{sp}}^{0} / M^{3,4} v s . C$ in MEK. Symbols are the same as in Figure 1.

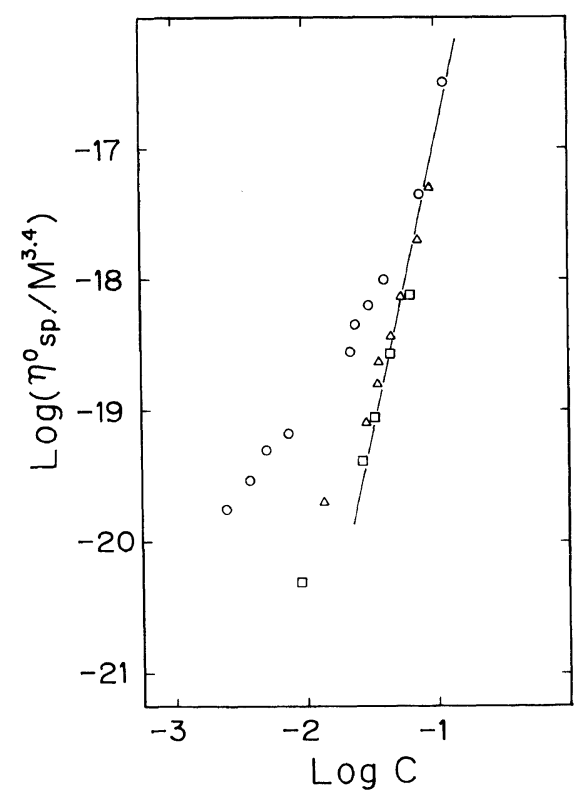

Figure 6. Double logarithmic plots of $\eta_{\mathrm{sp}}^{0} / M^{3,4} v s . C$ in benzene. Symbols are the same as in Figure 1.

respectively. In Figures $4-6$, the data in Figures $1-3$ are replotted in the double logarithmic form of $\eta_{\mathrm{sp}}^{0} / M^{3.4} v s$. $C$, where the data at the lower concentration were neglected since we were interested in the high concentra-

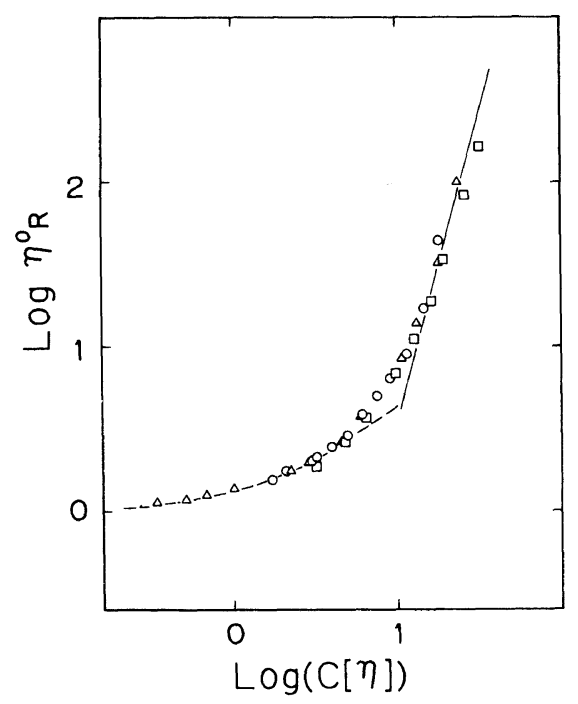

Figure 7. Double logarthmic plots of $\eta_{\mathbf{R}}^{0}$ vs. $C[\eta]$ in pyridine. Symbols are the same as in Figure 1. The broken curve and solid line denote eq 2 with $k^{\prime}=0.35$ and eq 3 with $v=0.58$, respectively.

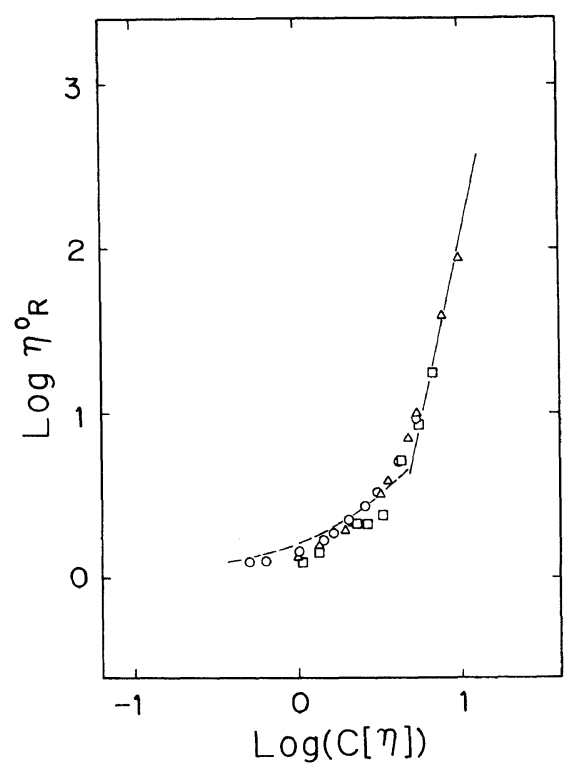

Figure 8. Double logarithmic plots of $\eta_{\mathrm{R}}^{\mathrm{O}}$ vs. $C[\eta]$ in MEK. Symbols are the same as in Figure 1. The broken curve and solid line denote eq 2 with $k^{\prime}=0.7$ and eq 3 with $v=0.53$, respectively.

tion region. These figures show that the data of $\eta_{\mathrm{sp}}^{0} / M^{3.4}$ converge on a straight line at high concentrations, and hence the 3.4 th power law 


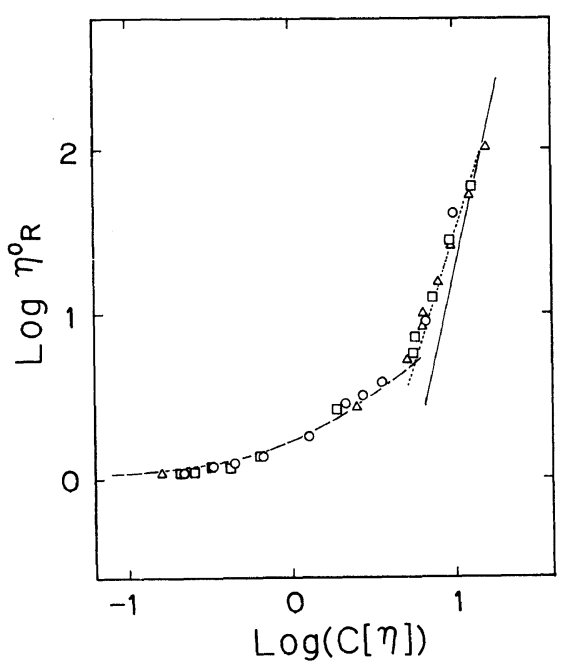

Figure 9. Double logarithmic plots of $\eta_{\mathrm{R}}^{0}$ vs. $C[\eta]$ in benzene. Symbols are the same as in Figure 1. The broken curve and solid line denote eq 2 with $k^{\prime}=0.7$ and eq 3 with $v=0.55$, respectively. The dotted line donotes eq 3 assuming $v=0.58$.

for $\eta^{0}$ of homopolymers in entangled regions also holds for the block copolymer solutions where the concentration is high enough. Figures $7-9$ show the viscosity data replotted in the double logarithmic form of $\eta_{\mathrm{R}}^{0} v s . C[\eta]$. These figures show that $\eta_{\mathbf{R}}^{0}$ of a block copolymer is expressed as a universal function of $C[\eta]$ in both dilute and semidilute solutions in the same way as that of homopolymer. Moreover, $\eta_{\mathrm{R}}^{0}$ of the block copolymer in pyridine is in good agreement with that of PS in good solvents. ${ }^{6}$ This result is consistent with the fact that the reduced osmotic pressure of the SP block copolymer in pyridine is in good agreement with that of PS in a good solvent as will be reported elsewhere. ${ }^{16}$

Now, let us compare quantitatively the data with eqs 2 and 3 . In the dilute region, $\eta_{\mathrm{R}}^{0}$ is in good agreement with eq 2 as shown by the broken lines, if the values of $k^{\prime}$ are assumed to be 0.35 in pyridine and 0.7 in MEK and benzene. These values are in good agreement with those of homopolymers in good and poor solvents, respectively.

To compare the experimental data in semidilute solutions with eq 3 , we need the $v$ values in these solvents, which can be calculated by assuming $v=(a+1) / 3$ from the exponent $a$ in the MHS equation. Although they slightly differ from the actual $v$ values, the difference does not significantly affect the following discussion.

MHS equations in these solvents are given by $^{13}$

$[\eta]=9.8 \times 10^{-5} M_{w}{ }^{0.73}$ (in pyridine at $25^{\circ} \mathrm{C}$ )

$[\eta]=3.5 \times 10^{-4} M_{w}{ }^{0.59}$ (in MEK at $25^{\circ} \mathrm{C}$ )

$[\eta]=1.5 \times 10^{-4} M_{w}{ }^{0.67}$ (in benzene at $11.4^{\circ} \mathrm{C}$ )

The third relationship was obtained in the present work. The $v$ values calculated from the above relationships are $0.58,0.55$, and 0.53 in pyridine, benzene and MEK, respectively. The slopes of straight lines in Figures 7-9 were calculated from eq 3 using these $v$ values. The dependences of $\eta_{\mathrm{R}}^{0}$ on $C[\eta]$ in pyridine (a commonly good solvent) and MEK (a commonly poor solvent) agree with those predicted by eq 3 with $v=0.58$ and 0.53 , respectively, whereas the dependence of $\eta_{\mathbf{R}}^{0}$ on $C[\eta]$ in benzene (a selective solvent) is lower than that predicted by eq 3 with $v=0.55$.

The critical concentration $C_{\mathrm{c}}$ for the microphase separation depends on the molecular weight of block copolymer and temperature T. Hashimoto et al. presented the following experimental relationship among them. ${ }^{7}$

$$
C_{\mathrm{c}} \propto T M^{-1 / 2}
$$

This means that $C_{\mathrm{c}}$ increases with temperature and decreasing molecular weight. For the present block copolymer in a commonly good solvent, almost the same relationship was found, as will be reported elsewhere. ${ }^{17} \mathrm{By}$ using the relationship, $C_{\mathrm{c}}$ is found to be around $0.1 \mathrm{gcm}^{-3}$ for the highest molecular weight sample (SP-18) in pyridine. Since eq 4 appears to be valid regardless of solvent power ${ }^{7}$, we may assume that $C_{\mathrm{c}}$ in MEK and benzene is not significantly different from that in pyridine. 
Thus, a few data at high concentrations in Figures $7-9$ are considered to be above $C_{\mathrm{c}}$. However, Figure 7 reveals that eq 3 is valid even above $C_{\mathrm{c}}$ in pyridine. This implies that the microphase-separated structures are too weak to affect $\eta^{0}$ in the concentration region slightly higher than $C_{\mathrm{c}}$.

Although the dependence of $\eta_{\mathrm{R}}^{0}$ on $C[\eta]$ in benzene is not explained by eq 3 as mentioned above, it is in good agreement with eq 3 if we assume $v=0.58$ for PS in a good solvent as shown by the dotted line in Figure 9. Moreover, it is to be noted that the dependence of $\eta_{\mathrm{R}}^{0}$ on $C[\eta]$ in MEK also can be explained by eq 3 assuming $v=0.54$ for PS in MEK. Here, we do not show the calculated line because the two $v$ values, which are for the SP block copolymer in MEK and for PS in MEK, are so close that both calculated lines are almost the same. As mentioned above, $\eta^{0}$ in pyridine (a commonly good solvent) is explained by eq 3 . Thus, these results in the three solvents indicate that the dependence of $\eta_{\mathrm{R}}^{0}$ on $C[\eta]$ is determined by the exponent $v$ of block component which has the larger excluded volume, regardless of the solvent.

Finally, it is to be noted that the $C[\eta]$ values at the crossover from dilute to semidilute regions are about 4, 6 and 10 in MEK, benzene and pyridine, respectively, as shown in Figures 7-9. They increase with the mean solvent power for the block copolymer indicated by the exponent $v$. This result is the same as observed for homopolymer solutions. ${ }^{5}$

\section{REFERENCES}

1. P. G. de Gennes, "Scaling Concepts in Polymer Physics," Cornell University Press, Ithaca, New York, 1979.

2. I. Noda, in "Molecular Conformation and Dynamics of Macromolecules in Condensed Systems," $\mathrm{M}$. Nagasawa, Ed., Elsevier, Tokyo, 1988.

3. P. J. Flory, "Principles of Polymer Chemistry," Cornell University Press, Ithaca, New York, N. Y., 1953.

4. P. G. de Gennes, Macromolecules, 9, 587594 (1976).

5. Y. Takahashi, Y. Isono, I. Noda, and M. Nagasawa, Macromolecules, 18, 1002 (1985).

6. Y. Takahashi, I. Noda, and M. Nagasawa, Macromolecules, 18, 2220 (1985)

7. T. Hashimoto, M. Shibayama, and H. Kawai, Macromolecules, 16, 1093 (1983).

8. H. Watanabe, and T. Kotaka, Polym. J., 14, 739 (1982).

9. Y. Ohta, T. Kojima, T. Takigawa, and T. Masuda, J. Rheol., 31, 711 (1987).

10. T. Tanaka, M. Omoto, and H. Inagaki, Macromolecules, 12, 146 (1979).

11. T. Tanaka, M. Omoto, and H. Inagaki, J. Macromol. Sci., Phys., B17, 229 (1980).

12. L. Ionescu, C. Picot, R. Duplessix, M. Duval, H. Benoit, J. P. Lingelser, and Y. Gallot, J. Polym. Sci., Polym. Phys. Ed., 19, 1033 (1981).

13. Y. Matsushita, Y. Nakao, K. Shimizu, I. Noda, and M. Nagasawa, Macromolecules, 21, 2790 (1988).

14. Y. Matsushita, Y. Nakao, R. Saguchi, H. Choshi, and M. Nagasawa, Polym. J., 18, 493 (1986).

15. S. H. Maron, (I. M. Krieger, A. W. Sisko, J. Appl. Phys., 25, 971 (1954).

16. M. Kawai, Y. Matsushita, and I. Noda, in preparation.

17. Y. Takahashi, Y. Matsushita, I. Noda, A. I. Nakatani, H. Kim, and C. C. Han, in preparation. 